\title{
GENERATING A SPECIFIC CLASS OF METAPHORS
}

\author{
Mark Alan Jones ${ }^{1}$ \\ Department of Computer and Information Sciences \\ University of Delaware, Newark DE 19716 \\ Internet: jones@udel.edu
}

\section{Introduction}

Although some progress has been made in the area of metaphor understanding, little has been made in metaphor generation. Current solutions rely upon a rather direct encoding of alternatives. There is no computational theory that can account for metaphor generation from basic principles. Although generating all types of metaphors from basic principles is very difficult, there is a subset of metaphors that are prevalent in natural expressions and perhaps more amenable to computational approaches. We call these transparentlymotivated (T-M) metaphors (Jones and McCoy 1992). Interestingly, metaphors in general, as well as the class described here, can be used to achieve important textual goals (e.g., brevity, conceptual fit, focus, perspective).

Metaphorical expressions often reflect conceptual models which are the basis for how we understand the world. Mark Johnson (1987) has made some important observations about the building blocks of thought, most notably that they are tied closely to our bodily experience. Among the building blocks he has described are attraction, blockage and containment. Consider describing the purchase of shares of stock as, "I took $\$ 2500$ out of my money market account and put it into Exxon common stock." The speaker did not literally put money into the stock, but rather bought stock with the money. This metaphor is based on the simplifying concepts that represent investments as containers which can hold money. When we write and talk we automatically use non-literal expressions that reflect our common conceptual groundings. These lead to very natural and easily understood expressions because we (speaker and audience) share these common conceptual groundings.

\section{Transparently-Motivated Metaphor}

All metaphors have a mapping between two domains. The more literal domain is called the tenor domain, the less literal is the metaphorical domain. In the stock example these are the financial domain and the containment domain respectively. T-M metaphors are similar to conventional metaphors (Lakoff and Johnson 1980, Lakoff 1987) in that they are both based upon familiar conceptual motivations. However, conventional metaphors are also defined in contrast to novel and dead metaphors. This distinction appeals to

\footnotetext{
${ }^{1}$ This work is supported by Grant \#H133E80015 from the National Institute on Disability and Rehabilitation Research. Support has also been provided by The Nemours Foundation.
}

knowledge about the history of expressions. In this sense, $\mathrm{T}-\mathrm{M}$ metaphors are broader in scope, however, in other ways they are more constrained.

Several qualities show that the stock example above is transparently-motivated. It is based on the bodily grounding of containment. It conveys the verb-phrase action message of a purchase being conducted, which is more than merely the mapping from containment to purchase. This mapping is not even highlighted, rather it is merely used to convey the purchase message.

Consider two counterexamples. The expression, "Men are wolves" (Black 1962), is not a transparently-motivated metaphor. Most importantly, its meaning is primarily the mapping itself; the mapping is not employed transparently to yield another specific meaning.

Consider describing the starting of a business in terms of gardening. "You must plant the seed in fertile soil, give it plenty of water..." Such allegorical metaphors are not T-M because they do not have a referent from the tenor domain which remains unchanged by the metaphor (e.g., "shares of stock"). The scope of this work is further constrained to metaphors that convey a verb-phrase meaning. The bounds of T-M metaphors that primarily convey other meanings, such as reference, are less clear.

So, transparently-motivated metaphors: 1) Are based on universal groundings that are often linked to bodily experience; 2) Convey a message (via the mapping) that is something more than the mapping; 3) Are subtle in the way that they do not draw attention to themselves as blatant metaphors. In fact, at first glance, these metaphors are often not recognized as non-literal; 4) Retain a referent from the tenor domain.

\section{Uses of Metaphor}

Metaphor is not merely a device that adds a flowery flavor to text. Rather, metaphor can be harnessed to achieve textual goals that may be difficult to achieve with literal statements. A simple goal that metaphor can achieve is that of being concise. More complex uses are explained below.

\subsection{Conceptual Fit}

We noted that the building blocks of thought may lead us to speak metaphorically. It is reasonable to conclude that metaphors based on such building blocks are easier to understand because they reflect human conceptualizations.

Two observations are salient. People naturally 
tend to describe things in a more concrete manner, even when the issue at hand is rather abstract. Such behavior generally yields more natural and understandable text. This explains why expressions like, "grasping an idea" are common. Second, it is natural to talk about things in the light of basic building blocks of thought that are commonly shared by a community. Therefore, describing a stock transaction as putting a token in a container may be more intuitive to the audience.

\subsection{Focus of Attention}

The traditional view of focus of attention is that it is something that is accomplished syntactically, such as by making the desired focus the subject of the sentence (as can be done with a passive construction). However, further inspection reveals that there is a relationship between semantic types and level of focus.

Jones and McCoy (1992) show evidence for the intuitive proposition that the semantic types of words/concepts affect the perceived level of focus attributed to those words/concepts. We introduce a simple focus hierarchy to model the effects of semantic types on focus levels. Items at the top of the focus hierarchy, because of their semantic qualities, are more likely to be focused upon than those below. Generally, concepts which are very concrete and volitional are toward the top while more amorphous and abstract things are below.

Given the focus hierarchy which explains inherent focus level according to the semantic type of an object, it is interesting to note that one effect of metaphorical statements can be to alter the perceived semantic type of an object (and therefore potentially raise the perceived focus level). Consider the metaphorical statement "AI is no stranger to object-oriented paradigms" (Elliot 1991). Notice that the phrase "is no stranger" has the effect of conceptaally personifying the objects involved (i.e., AI and object-oriented paradigms) since it is a phrase that, literally, can only be used with humans (or perhaps other animate objects). Compare the perceived focus level with that in a more literal rendition of the sentence such as: "AI and object-oriented paradigms have previously been incorporated together."

In the traditional view of focus of attention a word is treated as having a static semantics. However metaphor can make the semantic type of objects more flexible. By using a verb that only applies to humans, as above, the objects are pushed up the focus hierarchy towards the position that humans occupy.

\subsection{Perspective}

While the notion of perspective on an item is related to focus, they are distinct. Rather than concentrating on which object is focused on, perspective has to do with how an object is viewed. A given perspective on an item causes certain aspects of that item to be highlighted (and not others) (McCoy 1989).
Consider a couple with young children attending a party with all of their children's paraphernalia in tow. One tells the other "It is time for us to pull up stakes." Here, the leaving is metaphorically described via the camping domain, where leaving is an involved process. This use of metaphor has highlighted or put a particular perspective on the leaving that emphasizes the work involved.

\section{Approach to a Solution}

T-M metaphors are a promising sub-class of metaphors in which to work, because they carry special requirements that restrict the possible search space from which they can be generated. We have begun preliminary work to specify the basic structures and methodologies that together can generate good metaphors. Input to the system has two parts. The first part is the literal statement of what should be expressed, in a formal form. For example, describe the leave role of the object party. The second part of the input to the system is a specification of the goal that the metaphor is to achieve.

\subsection{General Approach}

The idea behind the approach is to identify related domains of the tenor domain that are appropriate as metaphorical domains. Both the tenor and metaphorical domains share some roles that are defined by their common ancestor in the "isa" hierarchy. Specifically, we require that they share the role that is the focus of the metaphor (that aspect of an action which is being referred to metaphorically). We can identify an ancestor of the tenor domain from which the tenor domain inherits the role in question. The metaphorical domain also will share this ancestor.

In addition to sharing the common ancestor, a reasonable metaphorical domain must have the following qualities:

- Be universal, or considered very familiar (with respect to the user model). If the audience is ignorant of the metaphorical domain, there is little hope of the expression's success.

- Have the potential to achieve additional goals (e.g., focus).

- Have specialized lexical expressions in the metaphorical domain for the role being described. This is necessary because the lexical expression used to describe the role is the only information that conveys the mapping. This restriction would not apply to non-literal expressions that explicitly state the mapping. Without the specialized lexical expression, a T-M metaphor cannot be generated.

These specifications constrain what potential metaphorical domains will be considered. By limiting the candidate domains, the space and search time requirements will be held down.

Consider how we can generate the metaphorical expression conveying "leave the party," while 
at the same time emphasizing the effort that it takes to leave (as in section 3.3 ). A party can be described, via "is-a" links of the abstraction hierarchy, as a human process. Such a process can have a termination. For partying, leaving and saying goodbye to everyone can be considered part of the termination of this process.

After ascending the "is-a" hierarchy to the proper level of abstraction, where the key role (termination) is specified, we can search for a candidate metaphorical domain that shares this ancestor. There are several possible metaphorical domains that meet this criteria. To narrow this choice several considerations come into play.

One of these considerations is whether the possible metaphorical domains have specialized expressions available for the role that the metaphor involves (termination). Two possible metaphorical domains that meet this criteria are camping, with "pulling up stakes," and electrical equipment, with "pull the plug."

Both domains are potential candidates. However, now the constraints imposed by the goal of the metaphor must be considered: emphasize the complexity of the termination. Will an allusion to camping rhetorically make leaving the party appear more involved? Here we must appeal to more detailed knowledge about the termination of the camping experience. In this case we find that the termination of a camping experience is not trivial, it requires a moderate amount of work (compared to the party and electrical domains). Therefore the camping domain may be chosen. Other metaphorical goals (e.g., focus) will cause different reasoning to be done in this final stage.

\subsection{Specific Conceptual Mappings}

The previous approach may work well for some T-M metaphors, but notice there is a severe restriction on the relationship between the tenor and metaphorical domains - they must have the role involved in the expression in common. Here we discuss how the previous method can benefit from additional information, which can link two very different domains.

Recall from section 3.1 that conceptual fit is a motivation for metaphor generation. The method introduced here helps implement the principle that it is useful to describe things in terms of shared conceptual roots. If a system is to generate metaphors that follow from conceptual roots, those roots must be represented in the system. We will need metaphorical domain selection rules and related mapping information to capture the conceptual roots by reflecting such common metaphorical behaviors as those pointed to in Lakoff and Johnson's work (Lakoff and Johnson 1980, Lakoff 1987). Selection rules will encode such familiar patterns as "describe progress in terms of a vehicle moving toward a goal" and "describe securities in terms of containers for money."

Consider describing the progress with a publication or career, with the intention of being as
Table 1: Progress in terms of a moving vehicle

\begin{tabular}{ccc} 
Tenor Domain & & Met. Domain \\
\hline $\begin{array}{c}\text { progress } \\
\text { negative progress }\end{array}$ & $\ldots \ldots .$. & forward \\
no progress & $\ldots \ldots .$. & backward \\
unsatisfactory progress & $\ldots \ldots$. & still \\
\end{tabular}

intuitive as possible (conceptual fit). A rule encoding the notion "describe progress in terms of a vehicle moving toward a goal" would be triggered. Closely attached to this rule is information about how the mapping from tenor domain to metaphorical domain should relate. Such information would include the mappings in Table 1.

Notice that these expressions for progress in the domain of physical motion are natural and probably more frequent than the "literal" forms found on the left side of Table 1. This may be because people understand progress in terms of motion. In this way metaphor generation can yield a more conceptually appropriate expression, which may actually be easier to understand than its literal counterpart.

There is potential for abstracting the information in the table. Note that the moving object has some starting point, some goal and some points on its path. With time involved, it also has speed. With a sophisticated model of this behavior in the metaphorical domain available, the four mappings in Table 1 could be derived. Interestingly, a more general structure matched with reasoning in the metaphorical domains could derive other expressions. With the knowledge that energy is required to move objects, and given that a prototypical moving object is a car that runs on gas, we could hope to generate "My career is running out of gas" from general knowledge and principles.

\section{REFERENCES}

Max Black (1962). Models and Metaphors. Cornell University Press, Ithica, NY.

Lance B. Elliot (1991). The bandwagon blues. $A I$ Expert, 6(5):11-13.

Mark Johnson (1987). The Body in the Mind: The Bodily Basis of Reason and Imagination. University of Chicago press, Chicago, IL.

Mark A. Jones and Kathleen F. McCoy (1992). Transparently-Motivated Metaphor Generation. In R. Dale, E. Hovy, D. Rosner and O. Stock, eds., Aspects of Automated Natural Language Generation: The 6th International Workshop on Natural Language Generation Proceedings, Trento, Italy. 231-246.

George Lakoff (1987). Women, Fire and Dangerous Things What Categories Reveal About the Mind. University of Chicago Press, Chicago, IL.

George Lakoff and Mark Johnson (1980). Metaphors we live by. University of Chicago Press, Chicago, IL.

Kathleen F. McCoy (1989). Generating context sensitive responses to object-related misconceptions. Artificial Intelligence, 41:157-195. 